\title{
New insights into the association of mid-childhood macronutrient intake to pubertal development in adolescence using nutritional geometry
}

\author{
Hoi Lun Cheng ${ }^{1,2 *}$, David Raubenheimer ${ }^{3}$, Katharine Steinbeck ${ }^{1,2}$, Louise Baur ${ }^{2}$ and Sarah Garnett ${ }^{2,4}$ \\ ${ }^{1}$ Academic Department of Adolescent Medicine, The Children's Hospital at Westmead, Westmead, NSW 2145, Australia \\ ${ }^{2}$ Discipline of Child and Adolescent Health, Sydney Medical School, The University of Sydney, Westmead, NSW 2145, Australia \\ ${ }^{3}$ Charles Perkins Centre, The University of Sydney, Camperdown, NSW 2006, Australia \\ ${ }^{4}$ Institute of Endocrinology and Diabetes, The Children's Hospital at Westmead, Westmead, NSW 2145, Australia \\ (Submitted 13 August 2018 - Final revision received 7 May 2019 - Accepted 22 May 2019)
}

\begin{abstract}
Nutritional geometry $(\mathrm{NG})$ is a novel dietary analysis approach that considers nutrient balance, rather than single nutrient effects, on health and behaviour. Through NG, recent animal experiments have found that lifespan and reproduction are differentially altered by dietary macronutrient distribution. Epidemiological research using NG reports similar findings for human ageing. Yet, the relation of macronutrient balance to human reproduction, especially reproductive maturation, remains undefined. We studied the impact of childhood macronutrient intake on pubertal maturation, by applying NG to an Australian longitudinal adolescent dataset. Food records, collected at age 8 years from 142 pre-pubertal children (females, 92; males, 50), were analysed for absolute energy, percentage energy and energy-adjusted residuals from protein, carbohydrate and fat. Pubertal stage change (assessed at 8,13 and 15 years) was modelled to obtain individual mathematical estimates of pubertal timing and tempo. Timing of menarche was recorded. The association of macronutrients to pubertal timing/tempo was assessed via NG, involving generalised additive models and heat maps to aid interpretation. Results showed lower dietary protein (relative to carbohydrate and fat) in girls consistently predicted earlier pubertal timing and menarche, and was related to faster pubertal tempo (all $P<0 \cdot 05$ ). No significant associations were identified in boys for both timing and tempo. Results suggest a role of non-protein macronutrients in facilitating female maturation; corroborating feeding and reproductive behaviour patterns observed in earlier NG studies of primates. Application of NG to other adolescent datasets is required to confirm the present findings. Such work would advance understanding of how nutrient balance shapes human development and health.
\end{abstract}

Key words: Puberty: Adolescents: Sexual maturation: Nutrition: Geometric frameworks

Life history theory is an ecological and evolutionary framework grounded upon a central tenet that lifespan and reproduction trade-off against each other ${ }^{(1)}$. Through this theory, the strong morbidity and mortality risks associated with earlier timing of pubertal onset or menarche may be explained as consequence of attaining reproductive maturity at a younger age ${ }^{(1)}$. Earlier pubertal timing has, for many years, been recognised as a predictor of cardiometabolic disease, some hormone-related cancers and mortality ${ }^{(2)}$. More recently, a link between faster pubertal tempo and suboptimal mental wellbeing has been described $^{(3,4)}$, suggesting that a trade-off potentially exists for the timing as well as speed of sexual maturation.

Over the past decade, there has been emerging interest in understanding how life history trade-offs are impacted by dietary macronutrients. Such interest has risen from advances in dietary analysis via nutritional geometry (NG), which is a non-linear data-driven analytic approach that considers how nutrient balance (rather than independent nutrient effects) relates to an outcome variable by 'mapping' data on a multi-dimensional space. Animal experiments using NG have generally found that a higher intake of protein relative to carbohydrate promotes fecundity, whereas the reverse dietary pattern prolongs survival $^{(5-8)}$. Such findings align with large epidemiological datasets on human ageing that show reduced mortality risk with high-carbohydrate, low-protein intake using the same analytic methodology ${ }^{(9)}$. Studies of longevous populations around the world (e.g. Okinawans) also report dietary macronutrient ratios that mirror the lifespan-promoting diets in the animal experiments ${ }^{(10)}$. The impact of macronutrients on human reproduction is, by contrast, less well explored. However, there is some evidence to suggest that the facilitatory effect of higher-protein, lower-carbohydrate intake on reproduction, as shown in the

Abbreviations: B3, Tanner stage 3 for breast development in girls; G3, Tanner stage 3 for genital development in boys; NG, nutritional geometry; PE:NPE, protein energy:non-protein energy ratio.

* Corresponding author: H. L. Cheng, email helen.cheng@health.nsw.gov.au 
animal experiments, may hold true for pubertal maturation in humans.

Several cohort studies, primarily from the 1990s and mostly in girls $^{(11)}$, have reported that higher total and/or animal protein intake in childhood predicted earlier timing of pubertal events including height take-off, thelarche and menarche ${ }^{(12-16)}$. Yet, the overall evidence is equivocal because a number of other studies have failed to identify any macronutrient association with pubertal timing ${ }^{(17-21)}$. Conflicting evidence may, at least partly, be due to the way nutrient data have been analysed. Nutrition research has historically involved regression modelling, which is a method that requires the nature/shape of a relationship (typically linear) to be determined by the researcher and not the data. In many cases, the non-independence (or substitution effects) of nutrient variables are also handled inappropriately, which makes disentangling individual macronutrient influences and the macronutrient $v$. total energy effects challenging. NG may be useful for overcoming these challenges ${ }^{(22)}$, and may offer new insights into the role macronutrient balance plays in human pubertal development.

This study aimed to use NG to assess the impact of childhood macronutrient balance on subsequent timing and tempo of puberty in adolescence. We hypothesised that children consuming greater amounts of total and animal protein relative to carbohydrate would experience earlier and faster puberty development, irrespective of total energy intake and other key confounders known to influence diet and pubertal development.

\section{Experimental methods}

\section{Participants and overall study design}

The present research used data from the Nepean Longitudinal Study. This was a cohort study that ran from 1996 to 2004, and which recruited 436 children ( $>95 \%$ Caucasian) from an Australian birth cohort born between August 1989 and April 1990 in Western Sydney ${ }^{(23)}$. The primary objective of the Nepean study was to explore foetal and mid-childhood influences on body composition and metabolic risk in adolescence $^{(23)}$. Data collection involved three study visits at: (i) 7-8 years of age (referred to as age 8 years or mid-childhood from this point onwards); (ii) age 13 years; and (iii) age 15 years, which span the pubertal development period allowing for offtimed normal puberty. Each visit involved collection of growth, metabolic and demographic data; the most relevant of which are outlined below. The Nepean study was conducted according to the guidelines laid down in the Declaration of Helsinki and all procedures involving human participants were approved by the ethics committees of The Children's Hospital at Westmead (protocol nos. 95038 and 2001/095) and the Wentworth Area Health Service (protocol nos. 95/051 and 2001/65). Written informed consent was obtained from all parents, with verbal assent given by all adolescents.

To be included in the present analyses, participants must have had a plausible food record at age 8 years, and provided information on age at menarche or had suitable hormone and Tanner stage data to allow estimation of their pubertal trajectory (see below).

\section{Collection and handling of dietary and demographic data}

Dietary intake at age 8 years was assessed using parent-assisted 3-d food records. Parents were guided on recording their child's food and drink intake using a standardised form. Dietary supplements, cooking methods, brand names and recipes were documented where applicable. Food records were analysed with DIET/1 version 3.10 (Xyris Software), using the Australian NUTTAB 95 database ${ }^{(24)}$. Food records were assessed for plausibility as per McCrory et ll $^{(25)}$, a method which has been used previously in adolescents ${ }^{(26)}$. Records were excluded if reported energy intake fell outside the plausibility range of predicted energy requirement; calculated at 76.2-123.8 and 76.5-123.5\% for boys and girls, respectively. Plausible records were analysed for absolute energy ( $\mathrm{kJ}$ per d), percentage energy (\% kJ per d) and energy-adjusted residuals ${ }^{(27)}$ from carbohydrate, fat, total protein and animal protein. The ratio of protein energy to non-protein energy (PE:NPE) was calculated.

Socio-economic variables assessed at age 8 years were mother's education, mother's occupational status and family income. Mother's education was categorised as incomplete high school, completed high school and tertiary education. Employment status was categorised as professional, non-professional or home duties/student/unemployed. An occupational prestige score, a widely used metric at the time of data collection ${ }^{(28)}$, was calculated from employment data. Gross annual family income (including government pensions and allowances) was grouped based on categories in the 1991 national census: <AUD30 000, AUD30 000-49 999 or $\geq$ AUD50 000 .

\section{Anthropometric and body composition assessment}

At age 8 years, height (nearest $0 \cdot 1 \mathrm{~cm}$ ) was measured using a Harpenden stadiometer (Holtain Ltd). Weight (nearest $0 \cdot 1 \mathrm{~kg}$ ) was measured in minimal clothing using an electronic scale (Detecto Scales Inc.). Waist circumference (nearest $0.1 \mathrm{~cm}$ ) was measured at the narrowest point between the lower rib border and iliac crest using flexible steel tape ${ }^{(23)}$. Age- and sex-specific BMI $z$-scores (zBMI) were calculated ${ }^{(29)}$, with overweight and obesity defined using International Obesity Task Force cut-points ${ }^{(30)}$.

\section{Pubertal assessment}

At age 8 years, 254 of the 436 participants provided a fasting morning blood sample for plasma testosterone and oestradiol analyses via radioimmunoassay ${ }^{(31)}$. For the present study, we only included those who had a measured hormone level in the childhood range (laboratory specific cut-off of $\leq 1 \mathrm{nmol} / 1$ for male testosterone and $\leq 56 \mathrm{pmol} / \mathrm{l}$ for female oestradiol), with which their pre-pubertal status could be verified. These participants were coded as being in Tanner stage 1 at age 8 years. During the age 13 and 15 year follow-ups, adolescents self-rated their Tanner stage using line drawings of female breast or male genital development ${ }^{(32)}$. Girls were asked if they had reached menarche, and if so, to recall the month and year of their first menstrual period. 


\section{Modelling of individual pubertal development trajectories}

Using a logistic mixed modelling procedure detailed in Marceau et $a l .{ }^{(33)}$, we described pubertal trajectories for each participant by modelling individual Tanner stage change against change in (fractional) age. To define the four parameter logistic curves with only three waves of data, a fourth pubertal time point had to be assigned for each individual which was done by coding Tanner stage 5 at age 17 years for girls and age 18 years for boys ${ }^{(34)}$. The decision to use this methodology over linear models of puberty was based on several factors. Firstly, logistic models describe the physiological process of maturation more realistically than linear trajectories, and have been shown to provide better modelfit $^{(3,33)}$. Secondly, logistic modelling considers within-person variation over time, unlike linear or duration-based measures of puberty that assume a constant rate of maturation. Thirdly, this methodology produces two model-derived scores for each individual, based on empirical Bayes estimates, which lend themselves naturally as mathematical estimates of pubertal timing and tempo. The score for pubertal timing represents the age at which a participant is halfway between the lower and upper asymptotes of the logistic curve; interpreted as the age at Tanner stage 3 for breast (B3) and genital (G3) development in girls and boys, respectively. The tempo score represents the slope of the logistic curve at the same halfway point, which gives the rate of maturation at mid-puberty expressed in Tanner stages per year. Model-derived scores were tested against the most reliable pubertal marker, i.e., menarcheal onset, using Spearman's correlation $\left(r_{\mathrm{s}}\right)$. Correlations between timing and tempo scores were assessed in both sexes.

\section{Statistical analyses}

Assessing selection bias. As only a subset of the Nepean cohort was included for the present study, we tested for selection bias by comparing birth weight, mid-childhood anthropometry and socio-economic variables between included and excluded participants using unpaired $t$ tests and Mann-Whitney $U$ tests. Normality was assessed via Shapiro-Wilk tests, with data presented as percentages, means and standard deviations or medians and interquartile ranges.

Nutritional geometry analysis of macronutrient balance v. pubertal outcomes. All analyses were undertaken across the absolute energy, percentage energy and energy-adjusted macronutrient variables. The balance of dietary carbohydrate, fat and protein in relation to each pubertal outcome (age at menarche; timing and tempo scores for breast/genital development) was assessed visually using a set of two-dimensional (2D) response surfaces, which plot the relationship of two nutrient predictors to a pubertal outcome (while holding the third nutrient axis at its median) using thin-plate splines ${ }^{(7)}$. Each response surface is read like a topographic map whereby areas in red denote the highest menarcheal age and timing/tempo scores, gradually transitioning to areas in blue denoting the lowest menarcheal age and timing/tempo scores. Importantly, interpretation of the timing and tempo results are reversed in that earlier age at menarche/ $\mathrm{B} 3 / \mathrm{G} 3$ is represented by areas in blue, whereas faster tempo of Tanner stage change is represented by areas in red. These response surfaces are summarised statistically using generalised additive models (GAM), which express the macronutrient relationships as main and interaction effects with respect to the outcome variable.

Using the same methodology of combining 2D response surfaces with GAM, we assessed the balance of total protein, animal protein and non-protein macronutrients on menarcheal age and the timing and tempo scores. An overall GAM was also constructed, which analysed the relationship of PE:NPE to each pubertal outcome while adjusting for total energy intake, birth weight, height, zBMI and mother's occupation prestige (other socio-economic variables were not included due to their categorical nature). All response surfaces and GAM were fitted using the fields and mgcv packages of the R language, respec-

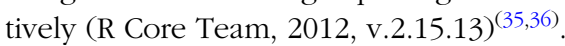

Linear regression analysis of macronutrient variables $\mathrm{v}$. pubertal outcomes. To aid comparison with prior research, we replicated the analytic strategy used in most existing studies through multiple regression modelling. Each regression model tested the main effects of the macronutrient variables on a pubertal outcome, adjusted for the same confounders described above. Collinearity between total energy intake and the macronutrient variables was tested by excluding total energy intake from the regression models, which did not alter any of the results. Linear regressions were performed on IBM SPSS Statistics 24 (IBM Corporation).

Justification of sample size. Sample size was determined by the availability of useable dietary and pubertal data from the Nepean cohort. Based on a two-sided significance level of 0.05 , a sample of ninety-two girls achieves $80 \%$ power to detect a minimal change in age at menarche and breast timing score of $0 \cdot 14$ year in a linear regression model with percentage protein as a predictor (SD of percentage protein: $2 \cdot 04$, SD of menarche residuals: 0.96; sD of breast timing score residuals: 0.99). A sample of fifty boys achieves $80 \%$ power to detect a change in genital timing score of $0 \cdot 11$ year in a linear regression model with percentage protein as a predictor (SD of percentage protein: $2 \cdot 17$; SD of residuals: 0.58). Calculations were performed using PASS 16 Power Analysis and Sample Size Software.

\section{Results}

\section{Participant characteristics}

Of the 436 children recruited to the Nepean study, 142 (ninetytwo girls, fifty boys) provided suitable dietary and pubertal data for NG analysis (online Supplementary Fig. S1). Upon assessing potential selection bias, the only observation was a slightly higher birth weight in the included $v$. excluded boys (by $190 \mathrm{~g}$; $P=0.051)$. No other differences in birth, mid-childhood or socio-economic variables were detected in either sex (Table 1).

Among participants included in the present analyses, birth weight and the mid-childhood variables were within normal range (Table 1). Overweight or obesity was prevalent in $21 \%$ of girls and $18 \%$ of boys, consistent with national rates at the time $^{(37)}$. Plasma oestradiol at 8 years was 18.8 (SD 4.2) $\mathrm{pmol} / 1$ in girls. Plasma testosterone at 8 years was $0 \cdot 70$ (interquartile 
Table 1. Participant birth weight, mid-childhood anthropometry and pubertal characteristics

(Numbers of subjects, mean values and standard deviations, percentages, and medians and interquartile ranges)

\begin{tabular}{|c|c|c|c|c|c|c|c|c|c|c|}
\hline \multirow[b]{3}{*}{ Participant characteristics } & \multicolumn{5}{|c|}{ Female } & \multicolumn{5}{|c|}{ Male } \\
\hline & \multicolumn{2}{|c|}{ Included } & \multicolumn{2}{|c|}{ Excluded } & \multirow[b]{2}{*}{$P$} & \multicolumn{2}{|c|}{ Included } & \multicolumn{2}{|c|}{ Excluded } & \multirow[b]{2}{*}{$P$} \\
\hline & Mean & SD & Mean & SD & & Mean & SD & Mean & SD & \\
\hline$n$ & \multicolumn{2}{|c|}{92} & \multicolumn{2}{|c|}{123} & & \multicolumn{2}{|c|}{50} & \multicolumn{2}{|c|}{171} & \\
\hline Birth weight (kg) & 3.37 & 0.50 & 3.36 & 0.49 & 0.927 & 3.66 & 0.56 & 3.50 & 0.50 & 0.051 \\
\hline \multicolumn{11}{|l|}{ Mother's education status (\%)* } \\
\hline Incomplete secondary education & \multicolumn{2}{|c|}{$50 \cdot 0$} & \multicolumn{2}{|c|}{$46 \cdot 3$} & 0.328 & \multicolumn{2}{|c|}{$50 \cdot 8$} & \multicolumn{2}{|c|}{$47 \cdot 1$} & 0.663 \\
\hline Completed secondary education & \multicolumn{2}{|c|}{$12 \cdot 0$} & \multicolumn{2}{|c|}{14.9} & & \multicolumn{2}{|c|}{$9 \cdot 2$} & \multirow{2}{*}{\multicolumn{2}{|c|}{7.4}} & \\
\hline Tertiary education & \multicolumn{2}{|c|}{38.0} & \multicolumn{2}{|c|}{38.8} & & \multicolumn{2}{|c|}{$40 \cdot 0$} & & & \\
\hline \multicolumn{11}{|l|}{ Mother's employment status (\%)* } \\
\hline Professional & \multicolumn{2}{|c|}{$13 \cdot 6$} & & & $0 \cdot 171$ & & & & & 0.704 \\
\hline Non-professional & & & & & & & & & & \\
\hline Home duties/student/unemployed & & & & & & & & & & \\
\hline Mother's occupational prestige score $\dagger$ & 4.90 & $1 \cdot 70$ & 4.85 & 1.48 & $0 \cdot 119$ & 4.90 & 1.95 & 4.80 & 1.70 & 0.338 \\
\hline Family income per annum (\%)* & & & & & & & & & & \\
\hline$<$ AUD30 000 & & & & & 0.664 & & & & & 0.439 \\
\hline AUD30 000-AUD49 999 & & & & & & & & & & \\
\hline$\geq$ AUD50 000 & & & & & & & & & & \\
\hline Anthropometry at age 8 years & & & & & & & & & & \\
\hline Height $(\mathrm{cm})$ & $127 \cdot 0$ & 5.9 & $126 \cdot 3$ & $6 \cdot 0$ & 0.418 & 128.0 & $6 \cdot 6$ & $127 \cdot 7$ & $6 \cdot 7$ & 0.749 \\
\hline Weight $(\mathrm{kg})$ & $27 \cdot 0$ & $5 \cdot 3$ & $27 \cdot 5$ & $6 \cdot 3$ & 0.596 & $26 \cdot 9$ & 4.4 & $27 \cdot 4$ & $6 \cdot 2$ & 0.591 \\
\hline Waist circumference $(\mathrm{cm}) \dagger$ & 54.9 & $6 \cdot 4$ & 55.5 & 7.4 & 0.550 & $56 \cdot 7$ & $5 \cdot 7$ & $56 \cdot 2$ & $6 \cdot 1$ & 0.942 \\
\hline BMI $z$-score & 0.19 & 1.08 & 0.40 & 0.95 & 0.129 & 0.19 & 0.88 & 0.21 & 1.07 & 0.880 \\
\hline Overweight (\%)* & & & & & 0.148 & & & & & 0.648 \\
\hline Obese $(\%)^{*}$ & & & & & & & & & & \\
\hline Pubertal characteristics & & & & & & & & & & \\
\hline Testosterone at age 8 years $(\mathrm{nmol} / \mathrm{l}) \dagger$ & - & - & - & - & - & 0.70 & 0.30 & - & - & - \\
\hline Oestradiol at age 8 years $(\mathrm{pmol} / \mathrm{l})$ & $18 \cdot 8$ & $4 \cdot 2$ & - & - & - & - & - & - & - & - \\
\hline Age at menarche (years) & $12 \cdot 6$ & 1.0 & - & - & - & - & - & - & - & - \\
\hline Puberty timing score (age in years)†‡ & $13 \cdot 2$ & 1.1 & - & - & - & 13.4 & 0.9 & - & - & - \\
\hline Puberty tempo score (stages per year) $\ddagger \S$ & $1 \cdot 15$ & 0.68 & - & - & - & 0.98 & 0.14 & - & - & - \\
\hline
\end{tabular}

* Data presented as a percentage.

† Data presented as median and interquartile range.

$\ddagger$ Derived from breast and genital development in girls and boys, respectively. Timing score is defined as age at Tanner stage 3 .

$\S$ Derived from breast and genital development in girls and boys, respectively. Tempo score is defined as the slope at Tanner stage 3 and is expressed in stages per year.

range $0 \cdot 30) \mathrm{nmol} / \mathrm{l}$ in boys. Age at menarche was $12 \cdot 6$ (SD 1.0) years. Median pubertal timing score (i.e. age at $\mathrm{B} / \mathrm{G} 3$ ) was 13.2 years in girls and 13.4 years in boys. Median pubertal tempo score (i.e. mid-pubertal rate of maturation) was 1.15 and 0.98 Tanner stages per year in girls and boys, respectively. A significant negative correlation was observed between timing and tempo scores in girls $\left(r_{\mathrm{s}}-0.40 ; P=0.001\right)$ but not boys (Fig. 1). The two measures of female pubertal timing, i.e. age at B3 and age at menarche, were significantly and positively correlated with each other $\left(r_{\mathrm{s}} 0 \cdot 29 ; P=0 \cdot 033\right.$; Fig. 1$)$.

\section{Macronutrient balance v. pubertal outcomes via nutritional geometry}

Generalised additive modelling (Table 2), together with associated diagnostic tests (online Supplementary Fig. S2), supported a significant association between macronutrient intake and menarcheal onset. Evaluation of response surfaces showed this association was not in the hypothesised direction, because lower absolute $(P=0.04)$ and energyadjusted $(P=0.03)$ protein were predictive of earlier menarche (Fig. 2). Other significant effects were detected for percentage fat $(P=0.009)$ and the interaction terms for absolute protein $\times$ carbohydrate $(P=0.005)$ and absolute protein $\times$ fat $(P=0.002)$, representing a higher intake of both non-protein macronutrients in earlier menstruating girls. Our overall model which adjusted for key confounders yielded similar findings, with lower PE:NPE strongly predicting earlier menarche $(P=0.008$; Table 2$)$. Animal protein intake did not show a significant relationship with menarcheal onset (data not shown).

Application of the NG procedure to breast development timing scores showed that lower protein intake predicted earlier age at B3 (Fig. 3). This observation was supported statistically by a significant main effect of absolute $(P=0.036)$ and percentage $(P=0.009)$ protein, and a non-significant trend for energyadjusted protein $(P=0 \cdot 070$; online Supplementary Table S1). Evaluation of tempo scores revealed a significant association for lower percentage protein $(P=0.046)$ and higher absolute carbohydrate intake $(P=0 \cdot 002)$ to faster breast development (online Supplementary Fig. S3). Adjustment for confounding in the overall models did not alter these findings, as lower PE: NPE remained significantly predictive of earlier age at B3 ( $P=0.006$; online Supplementary Table S1) and faster tempo $(P=0.031$; online Supplementary Table S2). Lower absolute $(P=0 \cdot 013)$, percentage $(P=0 \cdot 010)$ and energy-adjusted intake 
Table 2. Generalised additive models assessing macronutrient intake on age at menarche†

\begin{tabular}{|c|c|c|c|c|c|c|}
\hline Predictor variables & Estimated df & Ref. df & $F$ & $P$ & Deviance explained (\%) & AIC \\
\hline \multicolumn{7}{|c|}{ Absolute energy from macronutrients ( $k J$ per $d$ ) } \\
\hline $\mathrm{s}($ Protein) & $7.538 \mathrm{e}-01$ & 6 & 0.511 & $0.037^{\star}$ & \multirow[t]{7}{*}{23.2} & \multirow[t]{7}{*}{$222 \cdot 9$} \\
\hline $\mathrm{s}(\mathrm{CHO})$ & $1.496 \mathrm{e}-04$ & 6 & 0.000 & 0.403 & & \\
\hline $\mathrm{s}$ (Fat) & $1.092 \mathrm{e}-03$ & 6 & 0.000 & 0.236 & & \\
\hline $\mathrm{s}($ Protein, $\mathrm{CHO})$ & $2 \cdot 030 \mathrm{e}+00$ & 3 & 3.218 & $0.005^{\star *}$ & & \\
\hline $\mathrm{s}($ Protein, Fat) & $1.602 \mathrm{e}+00$ & 3 & 3.418 & $0.002^{* *}$ & & \\
\hline $\mathrm{s}(\mathrm{CHO}$, Fat $)$ & $3.687 e-05$ & 3 & 0.000 & 0.461 & & \\
\hline $\mathrm{s}($ Protein, $\mathrm{CHO}$, Fat $)$ & $6.050 e-05$ & 7 & 0.000 & 0.599 & & \\
\hline \multicolumn{7}{|c|}{ Percentage energy from macronutrients (\%) } \\
\hline $\mathrm{s}($ Protein) & $5 \cdot 634 \mathrm{e}-01$ & 6 & 0.215 & 0.110 & \multirow[t]{7}{*}{$11 \cdot 1$} & \multirow[t]{7}{*}{$230 \cdot 3$} \\
\hline $\mathrm{s}(\mathrm{CHO})$ & $4.644 \mathrm{e}-06$ & 6 & 0.000 & 0.794 & & \\
\hline $\mathrm{s}($ Fat $)$ & $8.072 e-01$ & 6 & 0.697 & $0.009^{* *}$ & & \\
\hline $\mathrm{s}($ Protein, $\mathrm{CHO})$ & $9.031 \mathrm{e}-01$ & 3 & 0.627 & 0.096 & & \\
\hline $\mathrm{s}($ Protein, Fat) & $5.093 e-06$ & 3 & 0.000 & 0.878 & & \\
\hline $\mathrm{s}(\mathrm{CHO}$, Fat $)$ & $3.373 e-06$ & 3 & 0.000 & 0.649 & & \\
\hline $\mathrm{s}($ Protein, $\mathrm{CHO}$, Fat $)$ & $1.419 \mathrm{e}-05$ & 7 & 0.000 & 1.000 & & \\
\hline \multicolumn{7}{|c|}{ Energy-adjusted macronutrient residuals } \\
\hline $\mathrm{s}($ Protein) & $7 \cdot 874 \mathrm{e}-01$ & 6 & 0.617 & $0.033^{*}$ & \multirow[t]{7}{*}{6.41} & \multirow[t]{7}{*}{$231 \cdot 6$} \\
\hline $\mathrm{s}(\mathrm{CHO})$ & $8 \cdot 686 \mathrm{e}-06$ & 6 & 0.000 & 0.686 & & \\
\hline $\mathrm{s}($ Fat $)$ & $3.676 \mathrm{e}-01$ & 6 & 0.081 & 0.252 & & \\
\hline $\mathrm{s}($ Protein, $\mathrm{CHO})$ & $7 \cdot 268 \mathrm{e}-05$ & 3 & 0.000 & 0.715 & & \\
\hline $\mathrm{s}$ (Protein, Fat) & $1.569 \mathrm{e}-06$ & 3 & 0.000 & 0.688 & & \\
\hline $\mathrm{s}(\mathrm{CHO}, \mathrm{Fat})$ & $3 \cdot 117 \mathrm{e}-05$ & 3 & 0.000 & 0.624 & & \\
\hline $\mathrm{s}($ Protein, $\mathrm{CHO}$, Fat) & $5 \cdot 865 e-06$ & 7 & 0.000 & 0.941 & & \\
\hline \multicolumn{7}{|c|}{ PE:NPE adjusted for total energy, birth weight, height, zBMI and mother's occupation } \\
\hline $\mathrm{s}(\mathrm{PE}: \mathrm{NPE})$ & $9 \cdot 230 \mathrm{e}-01$ & 6 & 1.997 & $<0.001^{\star \star \star}$ & \multirow[t]{6}{*}{$35 \cdot 6$} & \multirow[t]{6}{*}{$105 \cdot 1$} \\
\hline $\mathrm{s}($ Energylntake_kJ/d) & $1.877 \mathrm{e}-05$ & 6 & 0.000 & 0.753 & & \\
\hline $\mathrm{s}($ BirthWeight_kg) & $1.447 \mathrm{e}-05$ & 6 & 0.000 & 1.000 & & \\
\hline $\mathrm{s}($ Height_cm $)$ & $2 \cdot 745 \mathrm{e}-01$ & 6 & 0.063 & 0.247 & & \\
\hline $\mathrm{s}(\mathrm{zBMI})$ & $9 \cdot 064 \mathrm{e}-01$ & 6 & 1.613 & $0.002^{\star *}$ & & \\
\hline s(Occupation Score) $\ddagger$ & $5 \cdot 341 \mathrm{e}-05$ & 6 & 0.000 & 0.432 & & \\
\hline
\end{tabular}

Ref., reference; AIC, Akaike information criterion; $\mathrm{CHO}$, carbohydrate; PE:NPE, protein energy:non-protein energy ratio; zBMI, BMI $z$-score. ${ }^{*} P<0.05,{ }^{* *} P<0.01,{ }^{* * \star} P<0.001$.

$\dagger$ All models were fitted using the Gaussian distribution.

‡ Rank score for mother’s occupational prestige.
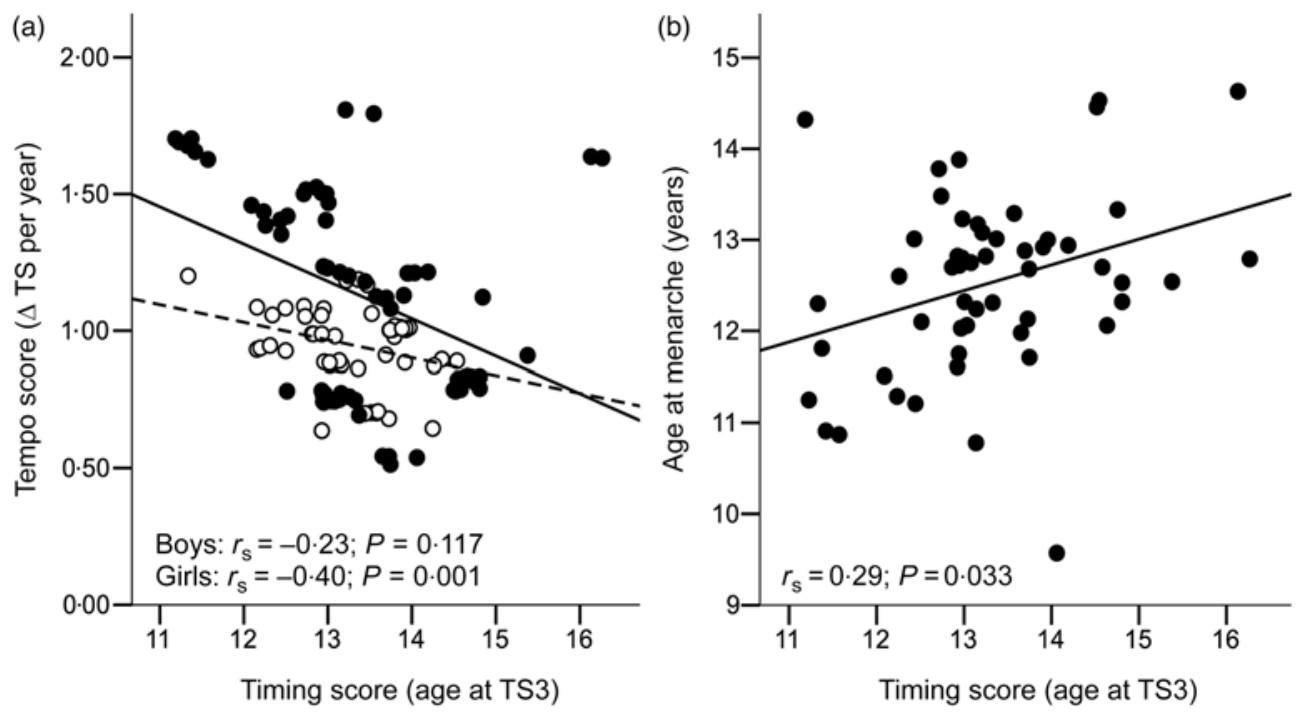

Fig. 1. Bivariate correlations for (a) pubertal timing and tempo scores in both sexes (-- - ---, boys; $\Delta$, Change; TS, Tanner stage; $r_{\mathrm{s}}$, Spearman's rho. 
(a)

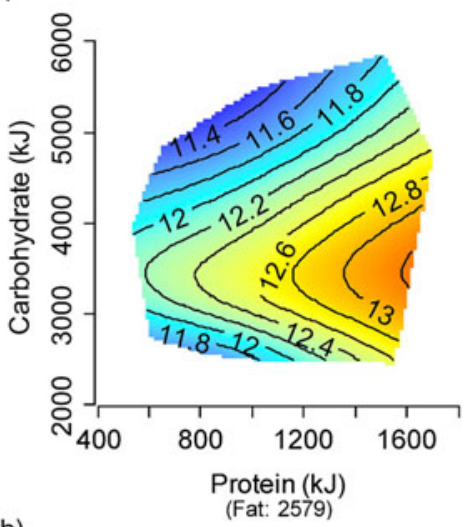

(b)

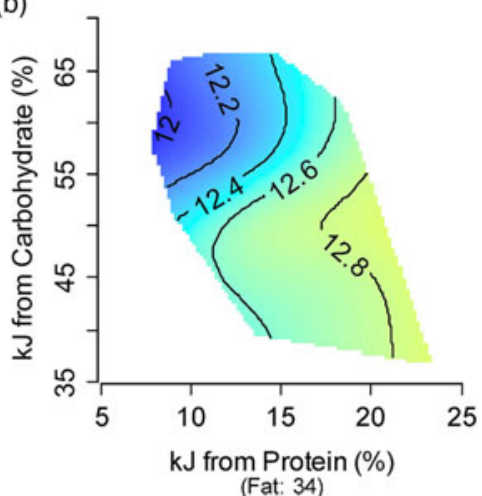

(c)
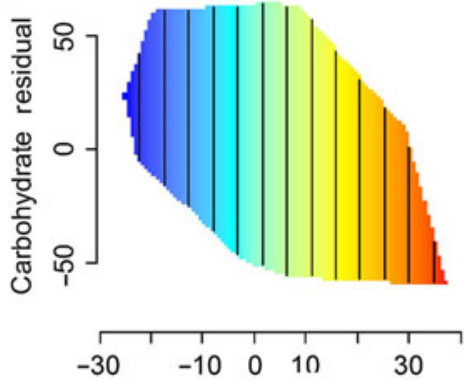

Protein residual

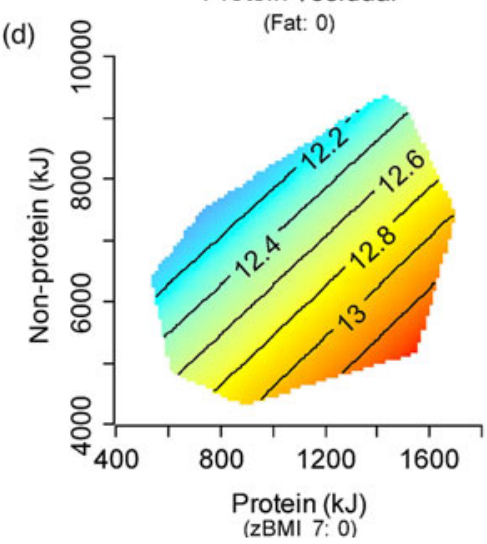

\section{Age at menarche (years)}
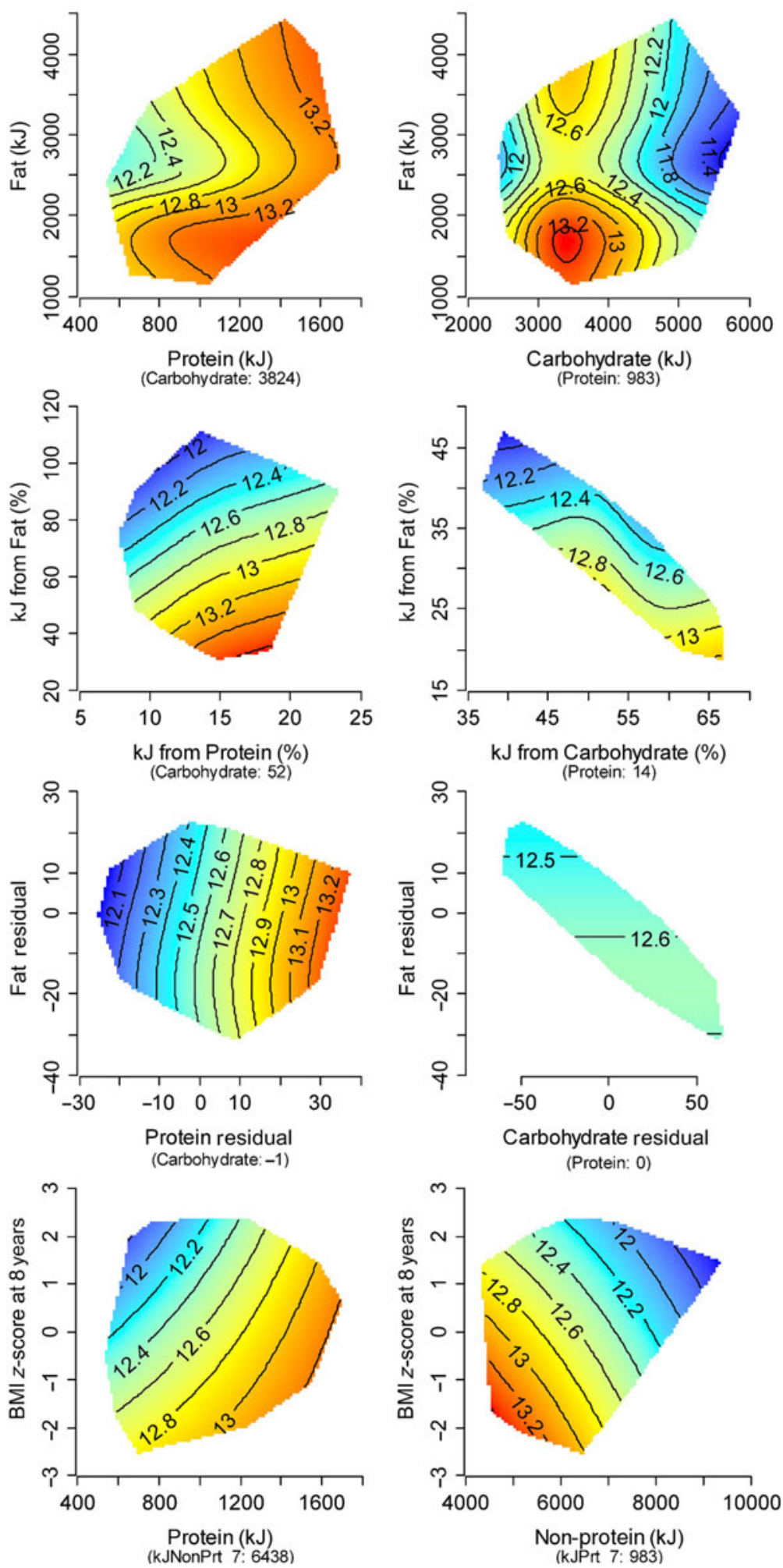

Fig. 2. Response surfaces showing the relationship between timing of menarche and: (a) absolute macronutrients (kJ/d); (b) percentage macronutrients (\% energy/d); (c) energy-adjusted macronutrient residuals; and (d) protein/non-protein intake (kJ/d) and BMl z-score (zBMI). Three two-dimensional (2D) slices are presented to show the interactive effects of all three macronutrients. In each $2 \mathrm{D}$ slice, the third factor is set at its median (shown in parentheses beneath the $x$-axis labels). Areas in blue represent the earliest maturers. Those with progressively later menarche are represented by the colour shift from blue to red. 


\section{Age at Tanner breast stage 3 (years)}
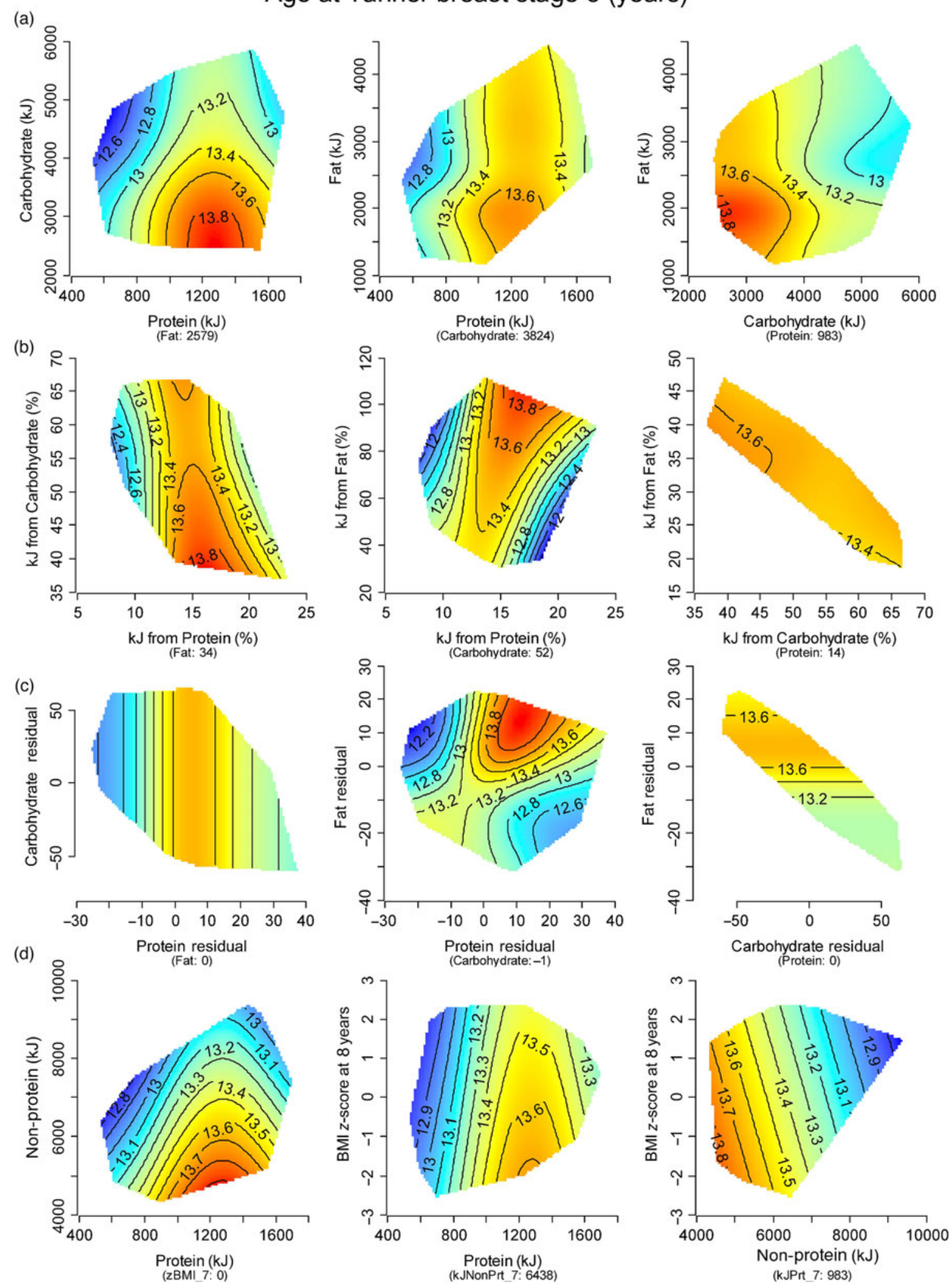

Fig. 3. Response surfaces showing the relationship between timing of breast development (age at Tanner stage B3) in girls and: (a) absolute macronutrients ( $\mathrm{JJ} / \mathrm{d})$; (b) percentage macronutrients (\% energy/d); (c) energy-adjusted macronutrient residuals; and (d) protein/non-protein intake (kJ/d) and BMI z-score. Three twodimensional (2D) slices are presented to show the interactive effects of all three macronutrients. In each $2 \mathrm{D}$ slice, the third factor is set at its median (shown in parentheses beneath the $x$-axis labels). Areas in blue represent the earliest maturers. Those with progressively later age at B3 are represented by the colour shift from blue to red. 
of animal protein was significantly associated with faster breast development tempo ( $P=0 \cdot 007$; online Supplementary Table S3 and Fig. S4).

No significant associations were detected between the macronutrient variables and timing (online Supplementary Table S4 and Fig. S5) and tempo (online Supplementary Table S5 and Fig. S6) scores for male genital development. Similarly, no significant animal protein effects were observed for male pubertal timing and tempo scores (data not shown).

\section{Macronutrient associations with pubertal outcomes via linear regression}

Linear models adjusted for confounding showed no significant main effects for the three macronutrients on age at menarche, or the timing and tempo scores for breast and genital development (online Supplementary Table S6). Additional testing of interaction terms (interactions were not assessed in prior studies) showed significant two- and three-way interactions for menarcheal age (protein $\times$ carbohydrate $\times$ fat: $P=0.037$ ) and age at B3 (protein $\times$ carbohydrate: $P=0.025$; protein $\times$ fat: $P=0.003$ ).

\section{Discussion}

In recent years, the growing use of NG in animal research has led to a paradigm shift in understanding how macronutrient intake influences lifespan and reproduction trade-offs. Application of this work to human data is in its infancy. As such, we sought to use NG to examine whether children consuming a higherprotein, lower-carbohydrate diet would experience an earlier and faster puberty. Our analyses in girls revealed associations that were contrary to our hypotheses. Furthermore, adjustment for confounding strengthened these unexpected results. Several potential explanations for our findings are discussed, which highlight clear knowledge gaps that remain around understanding nutrient balance with respect to human reproductive maturation.

Across various measures of macronutrient intake, our results consistently showed that a diet lower in protein, relative to both non-protein macronutrients, was predictive of earlier age at menarche and B3. A further finding was the association of lower total and animal protein to faster breast development, suggesting a potential impact of both macronutrient balance and protein source on pubertal tempo which has not been described previously. Overall, our observations align with the secular decrease in timing of thelarche and possibly menarche over the past century ${ }^{(38)}$, together with evidence of increasing protein dilution in the modern food supply ${ }^{(39)}$. Our data also support research describing a relationship between earlier menarche and excessive sugar-sweetened beverage consumption (protein intake was proportionately lower irrespective of BMI), which may be related to increased insulin secretion and gonadal/growth hormone bioavailability ${ }^{(40)}$. Nevertheless, we are aware our findings contradict several NG and non-NG studies, the results of which were used to formulate our hypothesis.

A number of studies using linear regression have reported an earlier growth spurt and menarche among children with higher total and/or animal protein intake (measured at various ages between 3 and 7 years ${ }^{(12-16)}$. Comparison of our Nepean cohort to these prior studies showed similar participant characteristics, research protocols and outcome variables, meaning that our discordant findings are most likely attributed to the mode of data analyses. Despite such evidence to support a facilitatory effect of protein on maturation, however, a substantial body of research has described null findings consistent with our regression analyses ${ }^{(17-21)}$. Significant two- and three-way interactions we observed also indicate some degree of non-linearity in the macronutrient-puberty relationship. This non-linearity was clearly illustrated through the NG methodology but not in the regression models.

To date, studies of macronutrient balance using NG have primarily been limited to animals. Earliest findings from Drosophila studies were largely contradictory to the present research ${ }^{(5,6)}$. Later experiments in rodents showed less definitive results, as some (uterine mass and ovarian follicles) but not all reproductive markers (number of oestrous cycles and recent ovulations) were maximised on a high dietary protein to carbohydrate ratio ${ }^{(8)}$. At present, the evidence most concordant with our work originates from non-human primates - a group of animals taxonomically similar to humans with comparable reproductive traits including menstrual cycle and gestation length and non-seasonal mating and births ${ }^{(41,42)}$. Observational studies of wild mountain gorillas have reported a strong dietary preference for fruit (a rich source of easily digestible sugars) when these are available above leaves (a rich source of protein $)^{(43,44)}$. Fruit availability, in turn, has consistently been shown across primate species to be a key determinant of fertility and population density ${ }^{(41,45)}$. Collectively, these lines of evidence indicate an important role of NPE in primate reproduction, lending support to the accelerated puberty we observed in our girls with lower PE:NPE. A number of caveats are, however, important to this interpretation. Firstly, fertility does not necessarily equate to reproductive maturation. Secondly, food shortages may be less relevant for humans (at least in developed societies) who have consistent access to all macronutrients throughout the year.

The present study represents one of three cohorts to have assessed macronutrient intake in relation to male puberty ${ }^{(12,13,46)}$. Historically, boys are under-represented in the literature due to the lack of a menarche-equivalent event that signifies achievement of reproductive competence. In our analyses, we modelled timing and tempo of self-reported genital change and found no association with the macronutrient variables. This result is in partial agreement with a German and US study that found higher dietary protein in childhood was consistently predictive of earlier height take-off and peak height velocity ${ }^{(12,46)}$, but not timing of voice-break $^{(12)}$. Height take-off and peak height velocity are, respectively, early (G2) and later (G3-4) pubertal events controlled by synergistic changes in growth and gonadal hormone levels ${ }^{(47,48)}$. This is in contrast to the voice-break which occurs at G3-4 and is primarily driven by the rise in testosterone ${ }^{(49)}$. Given our male timing measure (age at G3) is similar in both chronology and hormone regulation to voice-breaking, the null associations we observed may represent a true sex-related difference. Other limitations of small sample size, pubertal and dietary self-report and infrequent follow-up cannot be ruled out, which could also explain the lack of significant findings among boys. 
This study explored the use of NG in analysing relationships of macronutrient balance to pubertal maturation. NG has major strengths in that it provides an integrated approach to studying the complex nutrient interactions that are not captured by conventional regression methodologies. Other strengths of this study include multiple pubertal timing proxies in girls, and the use of non-linear models to describe puberty. The primary limitation of this study is the infrequent follow-ups, which potentially reduced our capacity to accurately describe pubertal trajectories within a logistic model framework. Despite this, our estimates for age at B3 were significantly correlated with age at menarche; the latter being our most reliable proxy for pubertal timing. These two proxies also yielded congruent findings, which add confidence to the validity of our model-derived scores as well as the new macronutrient associations identified for pubertal tempo. Other study limitations were the use of parental/ self-reported dietary and pubertal data (reporting bias is especially common in individuals with obesity ${ }^{(50,51)}$ ) and the absence of analyses testing different carbohydrate types. The decision to utilise self-reported Tanner stage was driven by ethical considerations precluding clinician staging in a healthy community sample.

In conclusion, this study identified an association between lower childhood protein intake and accelerated pubertal timing and tempo in girls, including earlier menarcheal onset. While this finding is in contrast to some older studies that described a facilitatory effect of protein on maturation, our data show parallels to animal research that have found NPE to be closely tied with primate reproduction. The absence of significant findings among boys reflects either sexual dimorphism in maturation or methodological limitations. Given this study is the first to explore pubertal development using the NG framework, our results strongly warrant replication in other adolescent datasets. The complex relationship between diet and biological processes, as demonstrated in this study, also argue for wider application of NG to contemporary research of human health and development.

\section{Acknowledgements}

We thank all participants and their families for generously donating their time to the study. Acknowledgements to Dr Frances Garden for her guidance on the statistical aspects of this project including assistance with sample size calculations.

The Nepean Longitudinal Study was supported by The Children's Hospital at Westmead Grant Research Scheme, the National Health and Medical Research Council of Australia (project grant no. 206501) and Meat and Livestock Australia. The above funders had no role in the design, analysis or writing of this article. The present study involves a secondary analysis of the Nepean study dataset, which was not supported by a specific grant from any funding agency, commercial or not-for-profit sectors. H. L. C. holds the Marie Bashir Clinical Research Fellowship in Adolescent Health, funded by the NSW Ministry of Health.

All authors designed the study; L. B. and S. G. collected the data; D. R. provided essential software for data analyses; H. L. C., D. R. and S. G. analysed the data; H. L. C., D. R. and S. G. wrote the article with critical feedback from K. S. and
L. B.; H. L. C. had primary responsibility for final content. All authors read and approved the final manuscript.

There were no conflicts of interest.

\section{Supplementary material}

To view supplementary material for this article, please visit https://doi.org/10.1017/S0007114519001326

\section{References}

1. Gluckman PD, Beedle AS, Hanson MA, et al. (2013) Human growth: evolutionary and life history perspectives. Nestle Nutr Inst Workshop Ser 71, 89-102.

2. Day FR, Elks CE, Murray A, et al. (2015) Puberty timing associated with diabetes, cardiovascular disease and also diverse health outcomes in men and women: the UK Biobank study. Sci Rep 5, 11208.

3. Beltz AM, Corley RP, Bricker JB, et al. (2014) Modeling pubertal timing and tempo and examining links to behavior problems. Dev Psychol 50, 2715-2726.

4. Mendle J (2014) Beyond pubertal timing: new directions for studying individual differences in development. Curr Dir Psychol Sci 23, 215-219.

5. Lee KP, Simpson SJ, Clissold FJ, et al. (2008) Lifespan and reproduction in Drosophila: new insights from nutritional geometry. Proc Natl Acad Sci U S A 105, 2498-2503.

6. Skorupa DA, Dervisefendic A, Zwiener J, et al. (2008) Dietary composition specifies consumption, obesity, and lifespan in Drosophila melanogaster. Aging Cell 7, 478-490.

7. Solon-Biet SM, McMahon AC, Ballard JW, et al. (2014) The ratio of macronutrients, not caloric intake, dictates cardiometabolic health, aging, and longevity in ad libitum-fed mice. Cell Metab 19, 418-430.

8. Solon-Biet SM, Walters KA, Simanainen UK, et al. (2015) Macronutrient balance, reproductive function, and lifespan in aging mice. Proc Natl Acad Sci U S A 112, 3481-3486.

9. Le Couteur DG, Solon-Biet S, Cogger VC, et al. (2016) The impact of low-protein high-carbohydrate diets on aging and lifespan. Cell Mol Life Sci 73, 1237-1252.

10. Le Couteur DG, Solon-Biet S, Wahl D, et al. (2016) New horizons: dietary protein, ageing and the Okinawan ratio. Age Ageing 45, 443-447.

11. Cheng G, Buyken AE, Shi L, et al. (2012) Beyond overweight: nutrition as an important lifestyle factor influencing timing of puberty. Nutr Rev 70, 133-152.

12. Gunther AL, Karaolis-Danckert N, Kroke A, et al. (2010) Dietary protein intake throughout childhood is associated with the timing of puberty. J Nutr 140, 565-571.

13. Remer T, Shi L, Buyken AE, et al. (2010) Prepubertal adrenarchal androgens and animal protein intake independently and differentially influence pubertal timing. J Clin Endocrinol Metab 95, 3002-3009.

14. Berkey CS, Gardner JD, Frazier AL, et al. (2000) Relation of childhood diet and body size to menarche and adolescent growth in girls. Am J Epidemiol 152, 446-452.

15. Rogers IS, Northstone K, Dunger DB, et al. (2010) Diet throughout childhood and age at menarche in a contemporary cohort of British girls. Public Health Nutr 13, 2052-2063.

16. Kissinger DG \& Sanchez PHA (1987) The association of dietary factors with the age of menarche. Nutr Res 7, 471-479.

17. Maclure M, Travis LB, Willett W, et al. (1991) A prospective cohort study of nutrient intake and age at menarche. Am J Clin Nutr 54, 649-656. 
18. Meyer F, Moisan J, Marcoux D, et al. (1990) Dietary and physical determinants of menarche. Epidemiology 1, 377-381.

19. Merzenich H, Boeing H \& Wahrendorf J (1993) Dietary fat and sports activity as determinants for age at menarche. Am J Epidemiol 138, 217-224.

20. Moisan J, Meyer F \& Gingras S (1990) Diet and age at menarche. Cancer Causes Control 1, 149-154.

21. Petridou E, Syrigou E, Toupadaki N, et al. (1996) Determinants of age at menarche as early life predictors of breast cancer risk. Int J Cancer 68, 193-198

22. Simpson SJ, Le Couteur DG \& Raubenheimer D (2015) Putting the balance back in diet. Cell 161, 18-23.

23. Garnett SP, Cowell CT, Baur LA, et al. (2001) Abdominal fat and birth size in healthy prepubertal children. Int JObes Relat Metab Disord 25, 1667-1673.

24. Lewis J, Milligan GC \& Hunt A (1995) NUTTAB95 Nutrient Data Table for Use in Australia. Canberra, Australia: Australian Government Publishing Service.

25. McCrory MA, McCrory MA, Hajduk CL, et al. (2002) Procedures for screening out inaccurate reports of dietary energy intake. Public Health Nutr 5, 873-882.

26. Huang TT, Roberts SB, Howarth NC, et al. (2005) Effect of screening out implausible energy intake reports on relationships between diet and BMI. Obes Res 13, 1205-1217.

27. Willett W \& Stampfer MJ (1986) Total energy intake: implications for epidemiologic analyses. Am J Epidemiol 124, 17-27.

28. Daniel A (1983) Power, Privilege and Prestige: Occupations in Australia. Melbourne: Longman Cheshire.

29. Kuczmarski RJ, Ogden CL, Guo SS, et al. (2002) 2000 CDC growth charts for the United States: methods and development. National Center for Health Statistics. Vital and Health Stat 11 246, 1-190.

30. Cole TJ, Bellizzi MC, Flegal KM, et al. (2000) Establishing a standard definition for child overweight and obesity worldwide: international survey. BMJ 320, 1240-1243.

31. Garnett SP, Hogler W, Blades B, et al. (2004) Relation between hormones and body composition, including bone, in prepubertal children. Am J Clin Nutr 80, 966-972.

32. Duke PM, Litt IF \& Gross RT (1980) Adolescents' selfassessment of sexual maturation. Pediatrics 66, 918-920.

33. Marceau K, Ram N, Houts RM, et al. (2011) Individual differences in boys' and girls' timing and tempo of puberty: modeling development with nonlinear growth models. Dev Psychol 47, 1389-1409.

34. Susman EJ, Houts RM, Steinberg L, et al. (2010) Longitudinal development of secondary sexual characteristics in girls and boys between ages $9 \frac{1}{2}$ and $15^{1 / 2}$ years. Arch Pediatr Adolesc Med 164, 166-173.

35. Wood SN (2006) Generalized Additive Models: An Introduction with R. Boca Raton, FL: CRC Press.

36. Wood SN (2003) Thin plate regression splines.J R Stat Soc Series B Stat Methodol 65, 95-114.
37. McLennan W \& Podger A (1998) 1995 National Nutrition Survey: Nutrient Intakes and Physical Measurements. Canberra: Australian Bureau of Statistics, Commonwealth of Australia.

38. Sorensen K, Mouritsen A, Aksglaede L, et al. (2012) Recent secular trends in pubertal timing: implications for evaluation and diagnosis of precocious puberty. Horm Res Paediatr 77, $137-145$.

39. Raubenheimer D, Machovsky-Capuska GE, Gosby AK, et al. (2015) Nutritional ecology of obesity: from humans to companion animals. Br J Nutr 113, S26-S39.

40. Carwile JL, Willett WC, Spiegelman D, et al. (2015) Sugar-sweetened beverage consumption and age at menarche in a prospective study of US girls. Hum Reprod 30, 675-683.

41. Knott CD, Emery Thompson M \& Wich SA (2009) Chapter 11: The ecology of female reproduction in wild orangutans. In Orangutans: Geographic Variation in Behavioral Ecology and Conservation, pp. 171-188 [SA Wich, SS Utami Atmoko, T Mitra Setia and CP van Schaik, editors]. New York: Oxford University Press.

42. Saltzman W, Tardify SD \& Rutherford JN (2010) Hormones and reproductive cycles in primates. In Hormones and Reproduction of Vertebrates, vol. 5: Mammals, pp. 291-327 [D Norris and K Lopez, editors]. New York: Academic Press.

43. Rothman JM, Raubenheimer D \& Chapman CA (2011) Nutritional geometry: gorillas prioritize non-protein energy while consuming surplus protein. Biol Lett 7, 847-849.

44. Hanya G \& Chapman CA (2013) Linking feeding ecology and population abundance: a review of food resource limitation on primates. Ecol Res 28, 183-190.

45. Hanya G, Stevenson P, van Noordwijk M, et al. (2011) Seasonality in fruit availability affects frugivorous primate biomass and species richness. Ecography 34, 1009-1017.

46. Alimujiang A, Colditz GA, Gardner JD, et al. (2018) Childhood diet and growth in boys in relation to timing of puberty and adult height: the longitudinal studies of child health and development. Cancer Causes Control 29, 915-926.

47. Marshall WA \& Tanner JM (1970) Variations in the pattern of pubertal changes in boys. Arch Dis Child 45, 13-23.

48. Tinggaard J, Mieritz MG, Sorensen K, et al. (2012) The physiology and timing of male puberty. Curr Opin Endocrinol Diabetes Obes 19, 197-203.

49. Harries ML, Walker JM, Williams DM, et al. (1997) Changes in the male voice at puberty. Arch Dis Child 77, 445-447.

50. Pietilainen KH, Korkeila M, Bogl LH, et al. (2010) Inaccuracies in food and physical activity diaries of obese subjects: complementary evidence from doubly labeled water and co-twin assessments. Int J Obes (Lond) 34, 437-445.

51. Shirtcliff EA, Dahl RE \& Pollak SD (2009) Pubertal development: correspondence between hormonal and physical development. Child Dev 80, 327-337. 